UDC 517.9

D. Blackmore (NJIT, Newark, USA),

A. K. Prykarpatsky (AGH Univ. Sci. and Technology Krakow, Poland and Ivan Franko State Ped. Univ. Drohobych, Ukraine),

E. Özçă̆, K. Soltanov (Hacettepe Univ. Ankara, Turkey)

\title{
INTEGRABILITY ANALYSIS
}

OF A TWO-COMPONENT BURGERS TYPE HIERARCHY

\section{АНАЛІЗ ІНТЕГРОВНОСТІ ДВОКОМПОНЕНТНОÏ ІЕРАРХІЇ ТИПУ БЮРГЕРСА}

The Lax integrability of a two-component polynomial Burgers type dynamical system is analyzed using a differentialalgebraic approach and its linear adjoint matrix Lax representation is constructed. A related recursive operator and an infinite hierarchy of nonlinear Lax integrable dynamical systems of the Burgers - Korteweg-de-Vries type are obtained by the gradient-holonomic technique. The corresponding Lax representations are presented.

Лаксівську інтегровність двокомпонентної поліноміальної динамічної системи типу Бюргерса проаналізовано за допомогою диференціально-алгебраїчного підходу. Побудовано ії лінійне спряжене матричне лаксівське зображення. Відповідний рекурсивний оператор та нескінченну ієрархію нелінійних динамічних систем типу БюргерсаКортевега-де Фріза, інтегровних за Лаксом, отримано за допомогою градієнтно-голономного методу. Наведено відповідні лаксівські зображення.

1. Introduction. Recently a great deal of research [16, 23, 24, 34, 37] has been devoted to classifying polynomial integrable dynamical systems on smooth functional manifolds. For example, in [37] one can find an extensive list of two-component polynomial dynamical systems of Burgers and Korteweg-de Vries type, which either reduce by means of a (in general nonlocal) change of variables to separable triangle Lax integrable forms or transform to completely linearizable flows. Amongst these systems, the authors of [37] singled out the following two-component Burgers type dynamical system

$$
\left(\begin{array}{l}
u_{t} \\
v_{t}
\end{array}\right)=K[u, v]:=\left(\begin{array}{c}
u_{x x}+2 u u_{x}+v_{x} \\
u_{x} v+u v_{x}
\end{array}\right)
$$

on a smooth Schwartz type functional manifold $M \subset C^{\infty}\left(\mathbb{R} ; \mathbb{R}^{2}\right)$, where $(u, v)^{\top} \in M$, the subscripts $x$ and $t$ denote, respectively, the partial derivatives with respect to the variables $x \in \mathbb{R}$ and $t \in \mathbb{R}_{+}$ and $t$ is the evolution parameter. Hereafter we will a priori assume that the dynamical system (1.1) possesses sufficiently smooth solutions in $t \in \mathbb{R}_{+}$, as it follows from the standard functional-analytic compactness principle considerations in [21].

It is mentioned in [37, p. 7706] that the Burgers type dynamical system (1.1) was extensively studied in $[8,22]$, where "... the symmetry integrability of $(1.1)$ as well as the existence of a recursive operator has already been demonstrated..." for it. The dynamical system (1.1) appears to have interesting applications, as its long-wave limit reduces to the well-known Leroux system [9, 38], describing dynamical processes in two-component hydro- and lattice gas dynamics. As claimed in 
[37, p. 7726], the integrability of (1.1) still remains unproven, and having found no relevant new result in the literature, we have decided to resolve this open problem by means of a combination of the gradient-holonomic [1, 31] method, linear adjoint mapping [30] techniques and recently devised $[2,27,28]$ differential-algebraic integrability tools. The following is our main result.

Theorem 1.1. The two-component polynomial Burgers dynamical system (1.1) possesses only two local conserved quantities $\int d x u$ and $\int d x v$ and no other infinite affine ordered local conserved quantities. Moreover, on the functional manifold $M$ the system (1.1) is linearizable by means of a Hopf-Cole type transformation and a dual adjoint mapping to the matrix Lax representation

$$
D_{x}\left(\begin{array}{l}
f \\
\hat{f}
\end{array}\right)=\left(\begin{array}{cc}
\left(u+\lambda^{-1} v-\lambda\right) / 2 & 0 \\
1 & \left.\left(\lambda-\lambda^{-1} v-u\right) / 2\right]
\end{array}\right)\left(\begin{array}{l}
f \\
\hat{f}
\end{array}\right)
$$

and

$$
D_{t}\left(\begin{array}{l}
f \\
\hat{f}
\end{array}\right)=\left(\begin{array}{cc}
\left.D_{x}(u+\lambda)\right] / 2+ & 0 \\
+(u+\lambda)\left(u+\lambda^{-1} v-\lambda\right) / 2 & \\
(u+\lambda) & \left.-D_{x}(u+\lambda)\right] / 2+ \\
& +(u+\lambda)\left(\lambda-u-\lambda^{-1} v\right) / 2
\end{array}\right)\left(\begin{array}{l}
f \\
\hat{f}
\end{array}\right)
$$

compatible for all $\lambda \in \mathbb{C} \backslash\{0\}$ with $(f, \hat{f})^{\top} \in \Lambda^{0}\left(\overline{\mathcal{K}}\left\{u, v ; D_{x}^{-1} \sigma \mid N\right\}\right)^{2}$, where $\overline{\mathcal{K}}\left\{u, v ; D_{x}^{-1} \sigma \mid N\right\}$ denotes $a[35]$ finitely extended differential ring $\overline{\mathcal{K}}\{u, v\}$. The related with the Lax operator (1.2) infinite hierarchy of generalized Burgers dynamical systems allows the following compact representation:

$$
D_{t_{n}}\left(u+\lambda^{-1} v-\lambda\right)=D_{x}\left[D_{x} \alpha_{n}(x ; \lambda)+\left(u+\lambda^{-1} v-\lambda\right) \alpha_{n}(x ; \lambda)\right],
$$

where the evolution parameters $t_{n} \in \mathbb{R}_{+}$and

$$
\alpha_{n}(x ; \lambda):=\left(\lambda^{n} \alpha_{n}(x ; \lambda)\right)_{+}
$$

for all natural $n \in \mathbb{N}$ is the corresponding nonnegative degree polynomial part generated by the asymptotic local functional solution $\alpha_{n}(x ; \lambda) \sim \sum_{j \in \mathbb{Z}_{+}} \lambda^{-j} \alpha_{j}[u, v]$ as $|\lambda| \rightarrow \infty$ to the differential functional equation

$$
D_{x}^{2} \alpha_{n}(x ; \lambda)+D_{x}\left(\left(u+\lambda^{-1} v-\lambda\right) \alpha_{n}(x ; \lambda)\right)=0 .
$$

As a simple consequence of the Theorem 1.1 one finds that the Burgers type dynamical system (1.1) does not admit a Hamiltonian formulation on the functional manifold $M$, and the corresponding recursive operator

$$
\Phi:=\left(\begin{array}{cc}
D_{x}+D_{x} u D_{x}^{-1} & 1 \\
D_{x} v D_{x}^{-1} & 0
\end{array}\right)
$$

satisfying the determining commutator equation

$$
D_{t} \Phi=\left[K^{\prime}, \Phi\right],
$$


for the two-component Burgers type dynamical system (1.1), introduced in [8, 22], cannot be factorized by compatible Poissonian structures, as they generally do not exist.

The scalar Lax representation (1.2) can be reduced by means of the nonlocal change of variables $\tilde{g}=f^{2} \exp \left[\lambda^{2} t-D_{x}^{-1}\left(\lambda^{-1} v-\lambda\right)\right] \in \Lambda^{0}\left(\overline{\mathcal{K}}\left\{u, v ; D_{x}^{-1} \sigma \mid N\right\}\right)$ to the simpler form

$$
D_{t} \tilde{g}=D_{x x} \tilde{g}+v \tilde{g}=0, \quad D_{x} \tilde{g}=u \tilde{g},
$$

compatible for $\tilde{g} \in \Lambda^{0}\left(\overline{\mathcal{K}}\left\{u, v ; D_{x}^{-1} \sigma \mid N\right\}\right)$ and not depending on the parameter $\lambda \in \mathbb{C} \backslash\{0\}$. The above representation (1.5) can be easily generalized to the following higher order evolution equation case:

$$
D_{t} \tilde{g}=D_{x}^{n} \tilde{g}+v \tilde{g}=0, \quad D_{x} \tilde{g}=u \tilde{g},
$$

where $n \in \mathbb{Z}_{+}$. Making use of the above nonlocal change of variables $\hat{g}=\tilde{g} \exp \left(\lambda^{-1} D_{x}^{-1} v\right) \epsilon$ $\in \Lambda^{0}\left(\overline{\mathcal{K}}\left\{u, v ; D_{x}^{-1} \sigma \mid N\right\}\right)$, one can obtain a new infinite hierarchy of two-component Lax integrable polynomial Burgers type dynamical systems, generalizing those discussed in [35, 36]. For instance, when $n=3$ we find the following dynamical Burgers type system of the third order:

$$
\begin{aligned}
& u_{t}=u_{3 x}+3\left(u u_{x}\right)_{x}+3 u^{2} u_{x}+v_{x}, \\
& v_{t}=(u r[u, v])_{x},
\end{aligned}
$$

where $r: J[u, v] \rightarrow C^{\infty}\left(\mathbb{R}^{2} ; \mathbb{R}^{2}\right)$ is a polynomial mapping on the jet-space $J\left(\mathbb{R}^{2} ; \mathbb{R}^{2}\right)$ of elements $\left(x, t ; u, v, D_{x} u, D_{x} v, D_{t} u, D_{t} v, D_{x}^{2} u, D_{x}^{2} v, \ldots\right) \in J\left(\mathbb{R}^{2} ; \mathbb{R}^{2}\right)$, suitably determined by the relationship (1.4) at $n=2$. Its scalar Lax representation is easily obtained from (1.2), (1.3) or from (1.6). The latter for $n=3$ easily gives rise to the scalar Lax representation

$$
\begin{gathered}
D_{x} \hat{g}=\left(u+\lambda^{-1} v\right) \hat{g} \\
D_{t} \hat{g}=D_{x x x} \hat{g}+3 \lambda^{-1} v D_{x x} \hat{g}+3\left(v_{x} / \lambda+v^{2} / \lambda^{2}\right) D_{x} \hat{g}+ \\
+\left(v_{x x} / \lambda+3 v v_{x} / \lambda^{2}+v^{3} / \lambda^{3}-(u \eta[u, v]) / \lambda\right) \hat{g}=0,
\end{gathered}
$$

compatible for all $\lambda \in \mathbb{C} \backslash\{0\}$ and $\hat{g} \in \Lambda^{0}\left(\overline{\mathcal{K}}\left\{u, v ; D_{x}^{-1} \sigma \mid N\right\}\right)^{2}$, which can be suitably extended by means of the related adjoint mapping to the matrix representation.

2. Differential-algebraic preliminaries. As our consideration of the integrability problem, discussed above, will be based on some differential-algebraic techniques, we need to include some additional differential-algebraic preliminaries $[1,11-15]$.

Take the ring $\mathcal{K}:=\mathbb{R}\{\{x, t\}\},(x, t) \in \mathbb{R} \times(0, T)$, of convergent germs of real-valued smooth functions from $C^{\infty}\left(\mathbb{R}^{2} ; \mathbb{R}\right)$ and construct the associated differential quotient ring $\mathcal{K}\{u, v\}:=$ $:=\operatorname{Quot}(\mathcal{K}[\Theta u, \Theta v])$ with respect to the functional variables $u, v \in \mathcal{K}$, where $\Theta$ denotes $[10$, $11,14,17,32]$ the standard monoid of all commuting differentiations $D_{x}$ and $D_{t}$, satisfying the standard Leibnitz condition, and defined by the natural conditions

$$
D_{x}(x)=1=D_{t}(t), \quad D_{t}(x)=0=D_{x}(t) .
$$

The ideal $I\{u, v\} \subset \mathcal{K}\{u, v\}$ is called differential if the condition $I\{u, v\}=\Theta I\{u, v\}$ holds. In the differential ring $\mathcal{K}\{u, v\}$, interpreted as an invariant differential ideal in $\mathcal{K}$, there are two naturally 
defined differentiations

$$
D_{t}, D_{x}: \mathcal{K}\{u, v\} \rightarrow \mathcal{K}\{u, v\},
$$

satisfying the commuting relationship

$$
\left[D_{t}, D_{x}\right]=0 .
$$

Consider the ring $\mathcal{K}\{u, v\}, u, v \in \mathcal{K}$, and the exterior differentiation $d: \mathcal{K}\{u, v\} \rightarrow \Lambda^{1}(\mathcal{K}\{u, v\})$, $d: \Lambda^{p}(\mathcal{K}\{u, v\}) \rightarrow \Lambda^{p+1}(\mathcal{K}\{u, v\})$ for $p \in \mathbb{Z}_{+}$, acting in the freely generated Grassmann algebras $\Lambda(\mathcal{K}\{u, v\})=\oplus_{p \in \mathbb{Z}_{+}} \Lambda^{p}(\mathcal{K}\{u, v\})$ over the field $\mathbb{C}$, where

$$
\begin{gathered}
\Lambda^{1}(\mathcal{K}\{u, v\}):=\mathcal{K}\{u, v\} d x+\mathcal{K}\{u, v\} d t+\sum_{j, k \in \mathbb{Z}_{+}} \mathcal{K}\{u, v\} d u^{(j, k)}+\sum_{j, k \in \mathbb{Z}_{+}} \mathcal{K}\{u, v\} d v^{(j, k),} \\
u^{(j, k)}:=D_{t}^{j} D_{x}^{k} u, \quad v^{(j, k)}:=D_{t}^{j} D_{x}^{k} v, \\
\Lambda^{2}(\mathcal{K}\{u, v\}):=\mathcal{K}\{u, v\} d \Lambda^{1}(\mathcal{K}\{u, v\}), \ldots, \Lambda^{p+1}(\mathcal{K}\{u, v\}):=\mathcal{K}\{u, v\} d \Lambda^{p}(\mathcal{K}\{u, v\}) .
\end{gathered}
$$

The triple $\mathcal{A}:=(\mathcal{K}\{u, v\}, \Lambda(\mathcal{K}\{u, v\}) ; d)$ will be called the Grassmann differential algebra with generatrices $u, v \in \mathcal{K}$. In the algebra $\mathcal{A}$, generated by $u, v \in \mathcal{K}$, one naturally defines the action of differentiations $D_{t}, D_{x}$ and $\partial / \partial u^{(j, k)}, \partial / \partial v^{(j, k)}: \mathcal{A} \rightarrow \mathcal{A}, j, k \in \mathbb{Z}_{+}$, as follows:

$$
\begin{gathered}
D_{t} u^{(j, k)}=u^{(j+1, k)}, \quad D_{x} u^{(j, k)}=u^{(j, k+1)}, \\
D_{t} v^{(j, k)}=v^{(j+1, k)}, \quad D_{x} v^{(j, k)}=v^{(j, k+1)}, \\
D_{t} d u^{(j, k)}=d u^{(j+1, k)}, \quad D_{x} d u^{(j, k)}=d u^{(j, k+1)}, \\
D_{t} d v^{(j, k)}=d v^{(j+1, k)}, \quad D_{x} d v^{(j, k)}=d v^{(j, k+1)}, \\
d P[u, v]=\sum_{j, k \in \mathbb{Z}_{+}} d u^{(j, k)} \wedge \partial P[u, v] / \partial u^{(j, k)}+\sum_{j, k \in \mathbb{Z}_{+}} d v^{(j, k)} \wedge \partial P[u, v] / \partial v^{(j, k)}= \\
=\sum_{j, k \in \mathbb{Z}_{+}}( \pm) \partial P[u, v] / \partial u^{(j, k)} \wedge d u^{(j, k)}+ \\
+\sum_{j, k \in \mathbb{Z}_{+}}( \pm) \partial P[u, v] / \partial v^{(j, k)} \wedge d v^{(j, k)}:=\left\langle P^{\prime}[u, v], \wedge(d u, d v)^{\top}\right\rangle_{\mathbb{R}^{2}},
\end{gathered}
$$

where the sign $\wedge$ denotes the standard [15] exterior multiplication in $\Lambda(\mathcal{K}\{u, v\})$, and for any $P[u, v] \in \Lambda(\mathcal{K}\{u, v\})$ the mapping

$$
P^{\prime}[u, v] \wedge: \Lambda^{0}(\mathcal{K}\{u, v\})^{2} \rightarrow \Lambda(\mathcal{K}\{u, v\}),
$$

is linear. Moreover, the commutation relationships

$$
D_{x} d=d D_{x}, \quad D_{t} d=d D_{t}
$$

hold in the Grassmann differential algebra $\mathcal{A}$. The following remark [14] is also important. 
Remark 2.1. Any Lie derivative $L_{V}: \mathcal{K}\{u, v\} \rightarrow \mathcal{K}\{u, v\}$, satisfying the condition $L_{V}: \mathcal{K} \subset \mathcal{K}$, can be uniquely extended to the differentiation $L_{V}: \mathcal{A} \rightarrow \mathcal{A}$, satisfying the commutation condition $L_{V} d=d L_{V}$.

The variational derivative, or the functional gradient $\nabla P[u, v] \in \Lambda(\mathcal{K}\{u, v\})^{2}$ with respect to the variables $u, v \in \mathcal{K}$, is defined for any $P[u, v] \in \Lambda(\mathcal{K}\{u, v\})$ by means of the following expression:

$$
\operatorname{grad} P[u, v]=P^{\prime, *}[u, v](1),
$$

where a mapping $P^{\prime, *}[u, v]: \Lambda^{0}(\mathcal{K}\{u, v\}) \rightarrow \Lambda^{0}(\mathcal{K}\{u, v\})^{2}$ is the formal adjoint mapping for that of (2.2). This is intrinsically based on the following important lemma, stated for a special case in $[10-14,26]$.

Lemma 2.1. Let the differentiations $D_{x}$ and $D_{t}: \Lambda(\mathcal{K}\{u, v\}) \rightarrow \Lambda(\mathcal{K}\{u, v\})$ satisfy the conditions (2.1). Then the mapping

$$
\begin{gathered}
\text { Ker grad } /(\operatorname{Im} d \oplus \mathbb{C}) \simeq H^{1}(\mathcal{A}):= \\
=\operatorname{Ker}\left\{d: \Lambda^{1}(\mathcal{K}\{u, v\}) \rightarrow \Lambda^{2}(\mathcal{K}\{u, v\})\right\} / d \Lambda^{0}(\mathcal{K}\{u, v\})
\end{gathered}
$$

is a canonical isomorphism, where $H^{1}(\mathcal{A})$ is the corresponding cohomology class of the Grassmann complex $\Lambda(\mathcal{K}\{u, v\})$.

It is well known [32] that for the differential ring $\mathcal{K}\{u, v\}$ not all of the cohomology classes $H^{j}(\mathcal{A}), j \in \mathbb{Z}_{+}$, are trivial. Nonetheless, one can impose on the functions $u, v \in \mathcal{K}$ some additional restrictions, which will give rise to the condition $H^{1}(\mathcal{A})=0$, or equivalently to the relationship $\operatorname{Ker} \nabla=\operatorname{Im} D_{x} \oplus \operatorname{Im} D_{t} \oplus \mathbb{C}$. In addition, the following simple relationship holds:

$$
\operatorname{grad}\left(\operatorname{Im} D_{x} \oplus \operatorname{Im} D_{t}\right)=0 .
$$

Using Lemma 2.1 one can define the equivalence class $\widetilde{\mathcal{A}}:=\mathcal{A} /\left\{\operatorname{Im} D_{x} \oplus \operatorname{Im} D_{t} \oplus \mathbb{R}\right\}:=$ $:=\mathcal{D}(\mathcal{A} ; d x d t)$, whose elements will be called functionals; that is, any element $\gamma \in \mathcal{D}(\mathcal{A} ; d x d t)$ can be represented as a suitably defined integral $\gamma:=\iint d x d t \gamma[u, v] \in \mathcal{D}(\mathcal{A} ; d x d t)$ for some $\gamma[u, v] \in \Lambda(\mathcal{K}\{u, v\})$ with respect to the Lebesgue measure $d x d t$ on $\mathbb{R}^{2}$.

Consider now our two-component dynamical system (1.1) as a polynomial differential constraint

$$
D_{t}(u, v)^{\top}=K[u, v]
$$

imposed on the ring $\mathcal{K}\{u, v\}$. The following definitions will be useful for our further analysis.

Definition 2.1. Let the reduced ring $\overline{\mathcal{K}}\{u, v\}:=\left.\mathcal{K}\{u, v\}\right|_{D_{t}(u, v)^{\top}=K[u, v]}$. Then the triple $\overline{\mathcal{A}}:=$ $:=(\overline{\mathcal{K}}\{u, v\}, \Lambda(\overline{\mathcal{K}}\{u, v\}), d)$ will be called a reduced Grassmann differential algebra over the reduced ring $\overline{\mathcal{K}}\{u, v\}$.

Definition 2.2. Any pair of elements $(\gamma[u, v], \rho[u, v])^{\top} \in \Lambda^{0}(\overline{\mathcal{K}}\{u, v\})^{2}$, satisfying the relationship

$$
D_{t} \gamma[u, v]+D_{x} \rho[u, v]=0,
$$

is called a scalar conserved quantity with respect to the differentiations $D_{x}$ and $D_{t}$. 
Using the differential-algebraic setting described above, one can naturally define the spaces of functionals $\mathcal{D}(\overline{\mathcal{A}} ; d x):=\overline{\mathcal{A}} /\left\{D_{x} \overline{\mathcal{A}}\right\}$ and $\mathcal{D}(\overline{\mathcal{A}} ; d t)=\overline{\mathcal{A}} /\left\{D_{t} \overline{\mathcal{A}}\right\}$ on the the reduced Grassmann differential algebra $\overline{\mathcal{A}}$. From the functional point of view these factor spaces $\mathcal{D}(\overline{\mathcal{A}} ; d x)$ and $\mathcal{D}(\overline{\mathcal{A}} ; d t)$ can be understood more classically as the corresponding spaces of suitably defined integral expressions subject to the measures $d x$ and $d t$, respectively. Then the relationship (2.4) means that the functional $\gamma:=\int d x \gamma[u, v] \in \mathcal{D}(\overline{\mathcal{A}} ; d x)$ is a conserved quantity for the differentiation $D_{t}$, and the functional $\Upsilon:=\int d t \rho[u, v] \in \mathcal{D}(\overline{\mathcal{A}} ; d t)$ is a conserved quantity for the differentiation $D_{x}$.

Since the differential relationship (2.3) naturally defines [14, 15] on the reduced ring $\overline{\mathcal{K}}\{u, v\}$ a smooth vector field $K: \overline{\mathcal{K}}\{u, v\} \rightarrow T(\overline{\mathcal{K}}\{u, v\})$, one can construct the corresponding Lie derivative $L_{K}: \overline{\mathcal{A}} \rightarrow \overline{\mathcal{A}}$ along this vector field and calculate the differential Lax [20] expression

$$
\partial \varphi[u, v] / \partial t+L_{K} \varphi[u, v]=0
$$

for the element $\varphi[u, v]:=\operatorname{grad} \gamma[u, v] \in \Lambda^{0}\left(\overline{\mathcal{K}}\left\{u, v ; D_{x}^{-1} \sigma \mid N\right\}\right)^{2}$, where $\overline{\mathcal{K}}\left\{u, v ; D_{x}^{-1} \sigma \mid N\right\}$ denotes some finitely extended differential ring $\overline{\mathcal{K}}\{u, v\}$ and $\gamma \in \mathcal{D}(\overline{\mathcal{A}} ; d x)$ is an arbitrary scalar conserved quantity with respect to the differentiation $D_{t}$. The following classical Noether-Lax lemma $[1,20$, 26, 31], inverse to the Lax relationship (2.5), holds.

Lemma 2.2 (E. Noether-P. Lax). Let a quantity $\varphi[u, v] \in \Lambda^{0}\left(\overline{\mathcal{K}}\left\{u, v ; D_{x}^{-1} \sigma \mid N\right\}\right)^{2}$ be such that the following equation:

$$
D_{t} \varphi[u, v]+K^{\prime, *}[u, v] \varphi[u, v]=0,
$$

equivalent to (2.5), holds in the ring $\overline{\mathcal{K}}\left\{u, v ; D_{x}^{-1} \sigma \mid N\right\}$ satisfying the differential constraint (2.3). Then, if the Volterra condition $\varphi^{\prime, *}[u, v]=\varphi^{\prime}[u, v]$ is satisfied in the ring $\overline{\mathcal{K}}\left\{u, v ; D_{x}^{-1} \sigma \mid N\right\}$, the constructed homology functional

$$
\gamma:=\int_{0}^{1} d \lambda \int d x\left\langle\varphi[\lambda u, \lambda v],(u, v)^{\top}\right\rangle_{\mathbb{C}^{2}} \in \mathcal{D}(\overline{\mathcal{A}} ; d x)
$$

is a scalar conserved quantity with respect to the differentiation $D_{t}$.

Assume now that the nonlinear two-component polynomial dynamical system (2.3) has a nontrivial compatible differential Lax representation in the form

$$
D_{x} f(x, t ; \lambda)=l[u, v ; \lambda] f(x, t ; \lambda), \quad D_{t} f(x, t ; \lambda)=p[u, v ; \lambda] f(x, t ; \lambda)
$$

for some matrices $l[u, v ; \lambda], p[u, v ; \lambda] \in \operatorname{End} \Lambda^{0}(\overline{\mathcal{K}}\{u, v\})^{q}, f(x, t ; \lambda) \in \Lambda^{0}\left(\overline{\mathcal{K}}\left\{u, v ; D_{x}^{-1} \sigma \mid N\right\}\right)^{q}$, analytically depending on a parameter $\lambda \in \mathbb{C}$, where $q \in \mathbb{Z}_{+} \backslash\{0,1\}$ is finite. Then the following important proposition, based on the gradient-holonomic approach, devised in [1,31], holds.

Proposition 2.1. The Lax integrable dynamical system (2.3) possesses a set (either finite or infinite) of naturally ordered functionally independent scalar conserved differential quantities

$$
D_{t} \sigma_{j}[u, v]+D_{x} \rho_{j}[u, v]=0,
$$

where the pairs $\left(\sigma_{j}[u, v], \rho_{j}[u, v]\right)^{\top} \in \Lambda^{0}(\overline{\mathcal{K}}\{u, v\})^{2}, j \in \mathbb{Z}_{+}$. 
Proof. Assume that the Lax integrable dynamical system (2.3) has a set (either finite or infinite) of naturally ordered functionally independent scalar conserved differential quantities (2.8). Let $\overline{\mathcal{K}}\left\{u, v ; D_{x}^{-1} \sigma \mid N\right\}$ denote the finitely extended differential ring $\mathcal{K}\left\{u, v ;\left\{D_{x}^{-1} \sigma_{j}[u, v]: 0 \leq j \leq N\right\}\right\}$ for an arbitrary integer $N \in \mathbb{Z}_{+}$under the constraints (2.3). Then the Lax equation (2.6), if considered on the invariant functional submanifold

$$
\begin{gathered}
M_{N}:=\left\{(u, v)^{\top} \in M: \operatorname{grad}\left\langle c^{(N)}, \int d x \Sigma^{(N)}\right\rangle_{\mathbb{C}^{N+1}}=0,\right. \\
\left.c^{(N)} \in \mathbb{C}^{N+1} \backslash\{0\}, \quad \Sigma^{(N)}:=\left(\sigma_{0}, \sigma_{1}, \ldots, \sigma_{N}\right)^{\top} \in \Lambda^{0}(\overline{\mathcal{K}}\{u, v\})^{N+1}\right\},
\end{gathered}
$$

allows [1, 31], as $|\lambda| \rightarrow \infty$, an asymptotic solution $\varphi(x ; \lambda) \in \Lambda^{0}\left(\overline{\mathcal{K}}\left\{u, v ; D_{x}^{-1} \sigma \mid N\right\}\right)^{2}$ in the form

$$
\varphi(x ; \lambda) \sim \psi(x, t ; \lambda) \exp \left\{\omega(x, t ; \lambda)+D_{x}^{-1} \sigma(x, t ; \lambda)\right\},
$$

with a scalar analytical "dispersion" function $\omega(x, t ; \cdot): \mathbb{C} \rightarrow \mathbb{C}$ determined for all $(x, t) \in \mathbb{R} \times[0, T)$, and the compatible local functional expansions

$$
\begin{aligned}
\Lambda^{0}(\overline{\mathcal{K}}\{u, v\})^{2} \ni \sigma(x, t ; \lambda) & \sim \sum_{j \in \mathbb{Z}_{+}} \sigma_{j}[u, v] \lambda^{-j+|\sigma|}, \\
\Lambda^{0}(\overline{\mathcal{K}}\{u, v\})^{2} \ni \psi(x, t ; \lambda) & \sim \sum_{j \in \mathbb{Z}_{+}} \psi_{j}[u, v] \lambda^{-j+|\psi|}
\end{aligned}
$$

for some fixed integers $|\sigma|,|\psi| \in \mathbb{Z}_{+}$. Moreover, owing to the Lax equation (2.6), all of the scalar functionals

$$
\gamma_{j}:=\int d x \sigma_{j}[u, v]
$$

for $j \in \mathbb{Z}_{+}$are conserved quantities with respect to the differentiation $D_{t}$. Now conversely, if the Lax equation (2.6) possesses an asymptotic, as $|\lambda| \rightarrow \infty$, solution in the form (2.9) $\varphi[u, v ; \lambda] \in$ $\in \Lambda^{0}\left(\overline{\mathcal{K}}\left\{u, v ; D_{x}^{-1} \sigma \mid N\right\}\right)^{2}$ with compatible expansions (2.10), then all of the scalar functionals (2.11) are, a priori, the conserved quantities with respect to the differentiation $D_{t}$. That is, there exist scalar quantities $\rho_{j}[u, v] \in \Lambda^{0}(\overline{\mathcal{K}}\{u, v\}), j \in \mathbb{Z}_{+}$, satisfying the relationships (2.8).

Proposition 2.1 is proved.

The analytical expressions for representation (2.9) and asymptotic expansions (2.10) for a Lax integrable dynamical system (2.3) follow readily from the general theory of asymptotic solutions [6, $33]$ to linear differential equations, applied to a linear differential system $(2.7)$ and from the following important fact $[1,7,25,31]$ : The trace functional $\Delta[u, v ; \lambda]:=\operatorname{tr}(F(x, t ; \lambda) C(\lambda) \bar{F}(x, t ; \lambda)) \in$ $\in \Lambda^{0}\left(\overline{\mathcal{K}}\left\{u, v ; D_{x}^{-1} \sigma \mid N\right\}\right)$ with any constant matrix $C(\lambda) \in$ End $\mathbb{C}^{q}$ is for almost all $\lambda \in \mathbb{C}$ a conserved quantity with respect to both differentiations $D_{t}$ and $D_{x}$, where $F(x, t ; \lambda)$ and $\bar{F}(x, t ; \lambda)$, $(x, t) \in \mathbb{R} \times \mathbb{R}_{+}$, are, respectively, the fundamental solutions to the linear Lax equation

$$
D_{x} f(x, t ; \lambda)=l[u, v ; \lambda] f(x, t ; \lambda)
$$

and its adjoint

$$
D_{x} \bar{f}(x, t ; \lambda)=-\bar{f}(x, t ; \lambda) l[u, v ; \lambda]
$$


where $f(x, t ; \lambda), \bar{f}^{\top}(x, t ; \lambda) \in \Lambda^{0}\left(\overline{\mathcal{K}}\left\{u, v ; D_{x}^{-1} \sigma \mid N\right\}\right)^{q}$. Consequently, the corresponding gradient

$$
\operatorname{grad} \Delta[u, v ; \lambda]:=\varphi[u, v ; \lambda] \in \Lambda^{0}\left(\overline{\mathcal{K}}\left\{u, v ; D_{x}^{-1} \sigma \mid N\right\}\right)^{2},
$$

owing to Lemma 2.2, a priori satisfies the Lax equation (2.6). Having assumed that $|\lambda| \rightarrow \infty$, from the asymptotic properties of linear equations (2.12) and (2.13) one obtains the result of Proposition 2.1.

3. Asymptotic, differential-algebraic and symplectic integrability analysis. We shall now analyze the Lax integrability of the two-component polynomial Burgers type dynamical system (1.1). In view of the approach described above, it is necessary to prove to the Lax equation (2.6) has an asymptotic solution of the form (2.9) in $\Lambda^{0}\left(\overline{\mathcal{K}}\left\{u, v ; D_{x}^{-1} \sigma \mid N\right\}\right)^{2}$. Concerning the dynamical system (1.1), the following proposition holds.

Proposition 3.1. The Lax equation (2.6) with the differential matrix operator

$$
K^{\prime, *}[u, v]=\left(\begin{array}{cc}
D_{x}^{2}-2 u D_{x} & -v D_{x} \\
-D_{x} & -u D_{x}
\end{array}\right),
$$

possesses the asymptotic solution

$$
\varphi(x ; \lambda)=(1,1 / \lambda)^{\top} g(x ; \lambda) \exp \left[-\lambda^{2} t-\lambda x+D_{x}^{-1}\left(u+\lambda^{-1} v\right)\right],
$$

as $|\lambda| \rightarrow \infty$, where the scalar invertible local functional element

$$
g(x ; \lambda):=\exp \left(-u+\sum_{j \in \mathbb{Z}_{+} \backslash\{0,1\}} D_{x}^{-1} \sigma_{j}[u, v] / \lambda^{j}\right) \in \Lambda^{0}(\overline{\mathcal{K}}\{u, v\}) .
$$

The solution (3.1) corresponds to the local conserved quantity $\Delta(\lambda):=\int d x\left(u+\lambda^{-1} v\right) \in \mathcal{D}(\overline{\mathcal{A}} ; d x)$ in the extended ring $\overline{\mathcal{K}}\left\{u, v ; D_{x}^{-1} \sigma \mid N\right\}^{2}$ :

$$
\operatorname{grad} \Delta(\lambda)[u, v]=\varphi(x ; \lambda) \in \Lambda^{0}\left(\overline{\mathcal{K}}\left\{u, v ; D_{x}^{-1} \sigma \mid N\right\}\right)^{2} .
$$

Proof. Assume that the Lax equation (2.6) possesses the asymptotic solution (2.9) as $|\lambda| \rightarrow \infty$, where $\omega(x, t ; \lambda)=-\lambda x-\lambda^{2} t$,

$$
\Lambda^{0}\left(\overline{\mathcal{K}}\left\{u, v ; D_{x}^{-1} \sigma \mid N\right\}\right)^{2} \ni \varphi(x ; \lambda)=\psi(x, t ; \lambda) \exp \left\{-\lambda^{2} t-\lambda x+D_{x}^{-1} \sigma(x, t ; \lambda)\right\}
$$

and

$$
\Lambda^{0}(\overline{\mathcal{K}}\{u, v\})^{2} \ni \psi(x, t ; \lambda)=(1, a(x, t ; \lambda))^{\top}
$$

which reduces to an equivalent system of the differential-functional relationships

$$
\begin{gathered}
D_{x}^{-1} \sigma_{t}-\lambda^{2}+\sigma_{x}+(-\lambda+\sigma)^{2}-(2 u+v a)(-\lambda+\sigma)-v a_{x}=0, \\
a_{t}+a\left(-\lambda^{2}+D_{x}^{-1} \sigma_{t}\right)-u a_{x}-(a u+1)(-\lambda+\sigma)=0 .
\end{gathered}
$$

The coefficients of the corresponding asymptotic expansions 


$$
\begin{aligned}
c \Lambda^{0}(\overline{\mathcal{K}}\{u, v\}) \ni a(x, t ; \lambda) & \sim \sum_{j \in \mathbb{Z}_{+}} a_{j}[u, v] \lambda^{-j}, \\
\Lambda^{0}(\overline{\mathcal{K}}\{u, v\}) \ni \sigma(x, t ; \lambda) & \sim \sum_{j \in \mathbb{Z}_{+}} \sigma_{j}[u, v] \lambda^{-j},
\end{aligned}
$$

should satisfy two infinite hierarchies of recurrent relationships

$$
\begin{gathered}
D_{x}^{-1} \sigma_{j-1, t}+\sigma_{j-1, x}+2 \sigma_{j}+\sum_{k \in \mathbb{Z}_{+}} \sigma_{j-1-k} \sigma_{k}-2 u \delta_{j-1,-1}-2 u \sigma_{j-1}- \\
-a_{j} v-v \sum_{k \in \mathbb{Z}_{+}} \sigma_{j-1-k} a_{k}-v a_{j-1, x}=0 \\
a_{j-2, t}-a_{j}+\sum_{k \in \mathbb{Z}_{+}} a_{j-2-k} D_{x}^{-1} \sigma_{k, t}-u a_{j-2, x}-\delta_{j-2,-1}- \\
-\sigma_{j-2}-u a_{j-1}-u \sum_{k \in \mathbb{Z}_{+}} \sigma_{j-2-k} a_{k}=0
\end{gathered}
$$

compatible for all $j \in \mathbb{Z}_{+}$. It is easy to calculate from (3.4) the corresponding coefficients

$$
\begin{gathered}
\sigma_{0}=u, \quad \sigma_{1}=u_{x}+v, \quad \sigma_{2}=v_{x}+u_{x x}+u u_{x}, \\
\sigma_{3}=D_{x}\left(u^{3} / 3+u v+u_{x x}+2 u u_{x}+v_{x}\right), \ldots, \sigma_{j}=D_{x}(\ldots), \ldots, \\
a_{0}=0, \quad a_{1}=1, \quad a_{2}=0, \quad a_{3}=0, \ldots, a_{j}=0, \ldots
\end{gathered}
$$

and thereby conclude that only two functionals

$$
\gamma_{0}:=\int d x \sigma_{0}[u, v]=\int d x u, \quad \gamma_{1}:=\int d x \sigma_{1}[u, v]=\int d x v,
$$

are nontrivial conservation laws with respect to the differentiation $D_{t}$, since all other functionals

$$
\gamma_{j}:=\int d x \sigma_{j}[u, v]=\int d x D_{x}(\ldots)=0
$$

are trivial in the ring $\overline{\mathcal{K}}\{u, v\}$. This also means that the gradient $\varphi(x ; \lambda):=\operatorname{grad} \int d x\left(u+\lambda^{-1} v\right)=$ $=(1,1 / \lambda)^{\top} \in \Lambda^{0}(\overline{\mathcal{K}}\{u, v\})^{2}$ satisfies the Lax equation (2.6) in the ring $\overline{\mathcal{K}}\{u, v\}$ and thus it should coincide with the expression (3.3). As a result it is easy to show that

$$
(1,1 / \lambda)^{\top}=(1,1 / \lambda)^{\top} g(x ; \lambda) \exp \left[-\lambda^{2} t-\lambda x+D_{x}^{-1}\left(u+\lambda^{-1} v\right)\right],
$$

where the scalar invertible element

$$
g(x ; \lambda):=\exp \left(-u+\sum_{j \in \mathbb{Z}_{+} \backslash\{0,1\}} D_{x}^{-1} \sigma_{j}[u, v] / \lambda^{j}\right) \in \Lambda^{0}(\overline{\mathcal{K}}\{u, v\}),
$$

ISSN 1027-3190. Укр. мат. журн., 2015, m. 67, № 2 
giving rise to the expressions (3.1) and (3.2).

Proposition 3.1 is proved.

As a consequence of Proposition 3.1, we can formulate the following result.

Theorem 3.1. The two-component polynomial Burgers type dynamical system (1.1) possesses only two local conserved quantities $\int d x u$ and $\int d x v$ and no other infinite affinely ordered conserved quantities (either local or nonlocal). Moreover, on the functional manifold $M$ the Burgers type dynamical system (1.1) is linearizable by means of a Hopf-Cole type transformation and the related linear adjoint mapping to the matrix Lax representation

$$
D_{x}\left(\begin{array}{l}
f \\
\hat{f}
\end{array}\right)=\left[\begin{array}{cc}
\left(u+\lambda^{-1} v-\lambda\right) / 2 & 0 \\
1 & \left.\left(\lambda-\lambda^{-1} v-u\right) / 2\right]
\end{array}\right]\left(\begin{array}{l}
f \\
\hat{f}
\end{array}\right),
$$

and

$$
\left(\begin{array}{l}
f \\
\hat{f}
\end{array}\right)=\left[\begin{array}{cc}
\left.D_{x}(u+\lambda)\right] / 2+ & 0 \\
+(u+\lambda)\left(u+\lambda^{-1} v-\lambda\right) / 2 & \\
(u+\lambda) & \left.-D_{x}(u+\lambda)\right] / 2+ \\
& +(u+\lambda)\left(\lambda-u-\lambda^{-1} v\right) / 2
\end{array}\right]\left(\begin{array}{l}
f \\
\hat{f}
\end{array}\right)
$$

compatible for all $\lambda \in \mathbb{C} \backslash\{0\}$, where vector function $(f, \hat{f})^{\top} \in \Lambda^{0}\left(\overline{\mathcal{K}}\left\{u, v ; D_{x}^{-1} \sigma \mid N\right\}\right)^{2}, N=2$.

Proof. It follows from Proposition 3.1 and recent results of [35] that one can apply to the twocomponent polynomial Burgers type dynamical system (1.1) the following generalized Hopf-Cole type linearizing transformation:

$$
u:=D_{x} \ln \tilde{g}(x ; \lambda)
$$

where

$$
\tilde{g}(x ; \lambda):=g(x ; \lambda)^{-1} \exp \left(\lambda^{2} t+\lambda x-\lambda^{-1} D_{x}^{-1} v\right) \in \Lambda^{0}\left(\overline{\mathcal{K}}\left\{u, v ; D_{x}^{-1} \sigma \mid N\right\}\right)
$$

and, by construction, it should be set $N=2$. Substituting (3.6) into (1.1), we obtain the system of linear equations

$$
D_{t} \tilde{g}=D_{x}^{2} \tilde{g}+v \tilde{g}, \quad D_{x} \tilde{g}=u \tilde{g},
$$

which clearly reduces to the following system of differential relationships:

$$
D_{t} \tilde{f}=\left(u_{x}+u^{2}+v\right) \tilde{f} / 2=0, \quad D_{x} \tilde{f}=(u / 2) \tilde{f},
$$

upon making the change of variables $\tilde{f}:=\tilde{g}^{1 / 2} \in \Lambda^{0}\left(\overline{\mathcal{K}}\left\{u, v ; D_{x}^{-1} \sigma \mid N\right\}\right)$. The system (3.8) can be specified further by making use of the substitution $\tilde{f}:=f \exp \left(\lambda x-\lambda^{-1} D_{x}^{-1} v\right)$, which giving rise to the scalar operator Lax representation

$$
D_{t} f=D_{x}(u+\lambda) f / 2+(u+\lambda) D_{x} f, \quad D_{x} f=\left(u+\lambda^{-1} v-\lambda\right) f / 2,
$$

compatible for all $\lambda \in \mathbb{C} \backslash\{0\}$.

Now we proceed to the construction of a suitably linearly extended adjoint differential relationships [30] for the system of equations (3.9). Using the standard method, it is easy to show that the following linearly adjoint relationship, compatible with the second equation of (3.9), 


$$
\begin{gathered}
D_{x} \hat{f}=D_{x}\left(\frac{\hat{f} f}{f}\right)=-f^{-1} \hat{f} D_{x} f+f^{-1} D_{x}(\hat{f} f)= \\
=-\left[\left(\lambda-\lambda^{-1} v-u\right) / 2\right] \hat{f}+f^{-1} D_{x}(\hat{f} f)=-\left[\left(\lambda-\lambda^{-1} v-u\right) / 2\right] \hat{f}+\chi[u, v ; \lambda] f,
\end{gathered}
$$

holds, where $D_{x}(\hat{f} f):=\chi[u, v ; \lambda] f^{2}$ for some arbitrarily chosen element $\chi[u, v ; \lambda] \in \overline{\mathcal{K}}\{u, v\}$. The compatibility of (3.10) with the first equation of (3.9) and its suitable extension gives rise to the condition $\chi[u, v ; \lambda]=1$. Thus, we have shown that the linearly adjoint relationship compatible with the second equation of (3.9) is

$$
D_{x} \hat{f}=-\left(\lambda-\lambda^{-1} v-u\right) \hat{f} / 2+f .
$$

The adjoint linear relationship compatible with the first equation of (3.9) is easily seen to be

$$
D_{t} \hat{f}=-D_{x}(u+\lambda) \hat{f} / 2+(u+\lambda) D_{x} \hat{f},
$$

and it is compatible with (3.11) for all $\lambda \in \mathbb{C} \backslash\{0\}$. It is now useful to rewrite equations (3.9), (3.11) and (3.12) as the following two equivalent matrix systems:

$$
D_{x}\left(\begin{array}{l}
f \\
\hat{f}
\end{array}\right)=\left(\begin{array}{cc}
\left(u+\lambda^{-1} v-\lambda\right) / 2 & 0 \\
1 & \left.\left(\lambda-\lambda^{-1} v-u\right) / 2\right]
\end{array}\right)\left(\begin{array}{l}
f \\
\hat{f}
\end{array}\right)
$$

and

$$
D_{t}\left(\begin{array}{l}
f \\
\hat{f}
\end{array}\right)=\left[\left(\begin{array}{cc}
\left.D_{x}(u+\lambda)\right] / 2+ & 0 \\
+(u+\lambda)\left(u+\lambda^{-1} v-\lambda\right) / 2 & \\
(u+\lambda) & \left.-D_{x}(u+\lambda)\right] / 2+ \\
& +(u+\lambda)\left(\lambda-u-\lambda^{-1} v\right) / 2
\end{array}\right)\right]
$$

where $(f, \hat{f})^{\top} \in \Lambda^{0}\left(\overline{\mathcal{K}}\left\{u, v ; D_{x}^{-1} \sigma \mid N\right\}\right)^{2}, N=2$. It follows from systems (3.13) and (3.14) that

$$
D_{x}(\hat{f} f)=f^{2}, \quad D_{t}(\hat{f} f)=(u+\lambda) f^{2} .
$$

Since the mutual compatibility condition of relationships (3.15) reduces to the expression

$$
D_{t} f=\left[D_{x}(u+\lambda) / 2\right] f+(u+\lambda) D_{x} f,
$$

exactly coinciding with the first equation of the system (3.14), we now can interpret both systems (3.13) and (3.14) as the corresponding matrix Lax representation for the Burgers system (1.1).

Theorem 3.1 is proved.

The scalar representation (3.7) can be easily generalized to the following higher order evolution equation:

$$
D_{t} \tilde{g}=D_{x}^{n} \tilde{g}+v \tilde{g}=0, \quad D_{x} \tilde{g}=u \tilde{g},
$$

where $n \in \mathbb{N} \backslash\{1,2\}, \tilde{g} \in \Lambda^{0}\left(\overline{\mathcal{K}}\left\{u, v ; D_{x}^{-1} \sigma \mid N\right\}\right)$ and no a priori constraint is imposed on the function $v \in \overline{\mathcal{K}}\{u, v\}$, except the functional $\int d x v \in \mathcal{D}(\overline{\mathcal{A}} ; d x)$ has to be a conserved quantity with respect to 
the differentiation $D_{t}$. Upon applying to (3.16) the nonlocal change of variables $u:=2 D_{x} \ln \tilde{f}[u, v ; \lambda]$ for $\tilde{f} \in \Lambda^{0}\left(\overline{\mathcal{K}}\left\{u, v ; D_{x}^{-1} \sigma \mid N\right\}\right), N=2$, one can obtain a new infinite hierarchy of two-component integrable polynomial Burgers type dynamical systems, generalizing the systems studied in [35, 36]. For instance, when $n=3$, we find the following dynamical Burgers-Korteweg-de Vries type dynamical system of the third order:

$$
\begin{aligned}
& u_{t}=u_{3 x}+3\left(u u_{x}\right)_{x}+3 u^{2} u_{x}+v_{x}, \\
& v_{t}=(u r[u, v])_{x}
\end{aligned}
$$

where $r[u, v] \in \overline{\mathcal{K}}\{u, v\}$ is for the present an arbitrary element. To choose those for which the dynamical systems of type (3.17) will possess suitably extended matrix Lax representations, it is natural to take the first pair of Lax type equations (3.5)

$$
\begin{aligned}
& D_{x} f=\left(u+\lambda^{-1} v-\lambda\right) f / 2, \\
& D_{x} \hat{f}=-\left(u+\lambda^{-1} v-\lambda\right) \hat{f} / 2+f
\end{aligned}
$$

for $(f, \hat{f})^{\top} \in \Lambda^{0}\left(\overline{\mathcal{K}}\left\{u, v ; D_{x}^{-1} \sigma \mid N\right\}\right)^{2}, N=2$, and to supplement it by means of the following systems of evolution equations, naturally generalizing that of (3.14) with respect to the temporal parameters $t_{n} \in \mathbb{R}$ :

$$
\begin{aligned}
& D_{t_{n}} f=D_{x} \alpha_{n}(x ; \lambda) f / 2+\alpha_{n}(x ; \lambda) D_{x} f, \\
& D_{t_{n}} \hat{f}=-D_{x} \alpha_{n}(x ; \lambda) \hat{f} / 2+\alpha_{n}(x ; \lambda) D_{x} \hat{f},
\end{aligned}
$$

which are, by construction, compatible for all $\lambda \in \mathbb{C} \backslash\{0\}$ for a polynomial in $\lambda, \alpha_{n}(x ; \lambda) \in \overline{\mathcal{K}}\{u, v\}$, $n \in \mathbb{N}$, satisfying the standard determining relationship

$$
D_{t_{n}}\left(u+\lambda^{-1} v-\lambda\right)=D_{x}\left[D_{x} \alpha_{n}(x ; \lambda)+\left(u+\lambda^{-1} v-\lambda\right) \alpha_{n}(x ; \lambda)\right] .
$$

It is also easy to check that for $n=1$ the choice

$$
\alpha_{1}(x ; \lambda)=u+\lambda
$$

yields the Burgers dynamical system (1.1).

The general algebraic structure of the whole infinite hierarchy of resulting dynamical systems (3.18) can be easily extracted from the matrix spectral Lax pair (3.13)

$$
D_{x}\left(\begin{array}{l}
f \\
\hat{f}
\end{array}\right)=\left(\begin{array}{cc}
\left(u+\lambda^{-1} v-\lambda\right) / 2 & 0 \\
1 & \left(\lambda-u-\lambda^{-1} v\right) / 2
\end{array}\right)\left(\begin{array}{l}
f \\
\hat{f}
\end{array}\right):=l[u, v ; \lambda]\left(\begin{array}{l}
f \\
\hat{f}
\end{array}\right),
$$

which allows, by means of the gradient-holonomic scheme [1,31], to obtain the commutator equation

$$
D_{x} S=[l, S], \quad S=\left(\begin{array}{cc}
S_{11} & S_{12} \\
S_{21} & S_{22}
\end{array}\right)
$$


for the related "monodromy" matrix $S:=S(x ; \lambda) \in \operatorname{sl}(2 ; \mathbb{C})$. This leads to the resulting $[25,31]$ canonical differential relationships for the gradient

$$
\left(\varphi_{1}, \varphi_{2}\right)^{\top}:=\varphi:=\operatorname{grad}(\operatorname{tr} S) \in \Lambda^{0}\left(\overline{\mathcal{K}}\left\{u, v ; D_{x}^{-1} \sigma \mid N\right\}\right)^{2}
$$

of the dynamical $D_{x}$ and $D_{t}$-invariant trace functional $\operatorname{tr} S(x ; \lambda) \in \mathcal{D}(\overline{\mathcal{A}} ; d x)$ :

$$
\begin{aligned}
& -D_{x} \varphi_{1}+D_{x}^{-1} u D_{x} \varphi_{1}+D_{x}^{-1} v D_{x} \varphi_{2}=\lambda \varphi_{1}, \\
& \varphi_{1}=\lambda \varphi_{2},
\end{aligned}
$$

where the component $\varphi_{1} \in \overline{\mathcal{K}}\{u, v\}$ has the following differential-algebraic representation:

$$
\varphi_{1}=\left(u+\lambda^{-1} v-\lambda\right) S_{21}+D_{x} S_{21}
$$

for some polynomial expression $S_{21}:=S_{21}(x ; \lambda) \in \overline{\mathcal{K}}\{u, v\}$. The differential expressions (3.20) can be rewritten in the useful matrix form:

$$
\Lambda \varphi=\lambda \varphi, \quad \Lambda:=\left(\begin{array}{cc}
-D_{x}+D_{x}^{-1} u D_{x} & D_{x}^{-1} v D_{x} \\
1 & 0
\end{array}\right)
$$

where the recursive operator $\Lambda: T^{*}(\overline{\mathcal{K}}\{u, v\}) \rightarrow T(\overline{\mathcal{K}}\{u, v\})$ satisfies the determining operator equation

$$
D_{t} \Lambda=\left[\Lambda, K^{\prime * *}\right]
$$

which follows directly from the Noether-Lax condition (2.6) and the adjoint linear spectral relationship (3.22).

Recall now that our Burgers type dynamical system (1.1) possesses only two conservations laws: $\gamma_{0}=\int d x u$ and $\gamma_{1}=\int d x v \in \mathcal{D}(\overline{\mathcal{A}} ; d x)$. This means that the expression (3.21) exactly equals $\varphi_{1}=\operatorname{grad}_{u} \gamma_{0}[u, v]=1$, or equivalently the condition

$$
D_{x}\left[D_{x} S_{21}(x ; \lambda)+\left(u+\lambda^{-1} v-\lambda\right) S_{21}(x ; \lambda)\right]=0
$$

should be satisfied for some element $S_{21}(x ; \lambda) \in \Lambda^{0}(\overline{\mathcal{K}}\{u, v\})$ and all $\lambda \in \mathbb{C} \backslash\{0\}$. The following proposition characterizes asymptotic solutions to (3.23) as $|\lambda| \rightarrow \infty$ and their relationships to the generalized dynamical systems (3.18).

Proposition 3.2. The nonnegative degree polynomial part of the asymptotic solution $S_{21}(x ; \lambda) \sim$ $\sim \sum_{j \in \mathbb{Z}_{+}} \lambda^{-j} S_{21}^{(j)}[u, v ; \lambda]($ as $|\lambda| \rightarrow \infty)$ to the differential relationship (3.23) makes it possible to represent the generating elements $\alpha_{n}(x ; \lambda) \in \Lambda^{0}(\overline{\mathcal{K}}\{u, v\})$ of the generalized dynamical systems (3.18) as

$$
\alpha_{n}(x ; \lambda)=\left(\lambda^{n} S_{21}(x ; \lambda)\right)_{+}
$$

for any $n \in \mathbb{Z}_{+}$.

Proof. Taking into account that the whole hierarchy of the generalized Burgers dynamical systems (3.18) can be represented in the recursive form

$$
D_{t_{n}}(u, v)^{\top}=\Phi^{n}\left(D_{x} u, D_{x} v\right)^{\top}, \quad \Phi:=\Lambda^{*}=\left(\begin{array}{cc}
D_{x}+D_{x} u D_{x}^{-1} & 1 \\
D_{x} v D_{x}^{-1} & 0
\end{array}\right)
$$


we can rewrite it as

$$
D_{t_{n}}\left(u+\lambda^{-1} v-\lambda\right)=D_{x}\left[D_{x} \alpha_{n}(x ; \lambda)+\left(u+\lambda^{-1} v-\lambda\right) \alpha_{n}(x ; \lambda)\right] .
$$

Here

$$
\alpha_{n}(x ; \lambda):=\left(\lambda^{n} \alpha(x ; \lambda)\right)_{+}
$$

is the corresponding nonnegative degree polynomial part generated by the asymptotic solution $\alpha(x ; \lambda) \sim \sum_{j \in \mathbb{Z}_{+}} \lambda^{-j} \alpha_{j}[u, v ; \lambda]$ as $|\lambda| \rightarrow \infty$ to the differential functional equation

$$
D_{x}^{2} \alpha(x ; \lambda)+D_{x}\left(\left(u+\lambda^{-1} v-\lambda\right) \alpha(x ; \lambda)\right)=0,
$$

which is equivalent to the dual to (3.22) symmetry relationship

$$
\Phi\left(D_{x} \alpha, D_{x} \beta\right)^{\top}=\lambda\left(D_{x} \alpha, D_{x} \beta\right)^{\top}
$$

with the generalized symmetry of the flow (1.1)

$$
\left(D_{x} \alpha, D_{x} \beta\right)^{\top}:=\sum_{j \in \mathbb{Z}_{+}} \lambda^{-j} \Phi^{j}\left(D_{x} u, D_{x} v\right)^{\top} .
$$

The observation that the differential functional equation (3.25) coincides with (3.23) proves the proposition.

The linear spectral problem (3.19) can be a priori generalized to the form

$$
D_{x}\left(\begin{array}{l}
f \\
\hat{f}
\end{array}\right)=\left(\begin{array}{cc}
\left(u+\lambda^{-1} v+\lambda\right) & h+\mu u_{x} \\
1 & \left(-\lambda-u-\lambda^{-1} v\right)
\end{array}\right)\left(\begin{array}{l}
f \\
\hat{f}
\end{array}\right),
$$

which at the constraint $v=0$ coincides with that for the Boussinesq-Burgers (at $\mu=0$ )

$$
\begin{aligned}
& u_{t}=-2 u u_{x}+h_{x} / 2, \\
& h_{t}=-2(u h)_{x}+u_{x x x} / 2,
\end{aligned}
$$

and with the Broer-Kaup-Kupershmidt (at $\mu=1$ )

$$
\begin{aligned}
& u_{t}=-u u_{x}+u_{x x}-h_{x}, \\
& h_{t}=-(u h)_{x}-h_{x x}
\end{aligned}
$$

hydrodynamic systems, whose integrability and soliton-like solutions were studied in $[4,18,19,31]$. The related multicomponent "dark"-type extensions of (3.26) and (3.27) were recently constructed in [5].

Theorem 1.1 also implies that the Burgers type dynamical system (1.1) does not admit a Hamiltonian formulation on the functional manifold $M$. This means that the recursive operator (3.22) constructed above and earlier in $[8,22]$ for the two-component Burgers type dynamical system (1.1) is not factorizable by means of suitably defined compatible Poissonian structures, as they on the whole, do not exist. This is strongly related to the fact that the dynamical system (1.1) does not possess an infinite hierarchy of local conservation laws, whose existence yields such a factorization. Nonetheless, similar to the situation in [35], if one shows that the Burgers type dynamical system (1.1) does possess another infinite hierarchy of nonlocal conservation laws, then to some degree the symmetry recursive operator (3.24) will already be factorized by means of the constructed Poissonian structures. But to our knowledge, this remains an open problem. 
4. Conclusion. Employing mainly the differential-algebraic approach [1, 27, 28, 31, 35] for testing the Lax integrability of nonlinear dynamical systems on functional manifolds, we proved that the two-component polynomial Burgers type dynamical system (1.1) has an adjoint matrix Lax representation and a corresponding recursive operator, which does not allow a bi-Poissonian factorization and so only makes it possible to construct two local conserved quantities. The problem of constructing a generalized bi-Poissonian factorization of a suitably powered recursive operator, similar to that in [35], is left for future analysis. Thus, the differential-algebraic approach, together with considerations based on the symplectic geometry, can serve as a simple, effective tool for analyzing the Lax integrability of a wide class of polynomial nonlinear dynamical systems on functional manifolds. Moreover, as was recently demonstrated in [29], this approach also appears to be useful in the case of nonlocal polynomial dynamical systems.

Acknowledgements. D. Blackmore acknowledges the support of the National Science Foundation (Grant CMMI-1029809), A. Prykarpatsky cordially thanks Prof. J. Cieślińskiemu (Białystok University, Poland), Prof. I. Mykytyuk (Pedagogical University of Krakow, Poland) and Prof. A. Augustynowicz (Gdansk University, Poland) for useful discussions of the results obtained. A. Prykarpatsky, E. Özçağ and K. Soltanov gratefully acknowledge partial support of the research in this paper from the Turkey-Ukrainian: TUBITAK-NASU Grant 110T558.

1. Blackmore D., Prykarpatsky A. K., Samoylenko V. H. Nonlinear dynamical systems of mathematical physics. - New York: World Sci. Publ., 2011.

2. Blackmore D., Prykarpatsky Y. A., Bogolubov N., Prykarpatski A. Integrability of and differential-algebraic structures for spatially 1D hydrodynamical systems of Riemann type // Chaos, Solitons and Fractals. - 2014. - 59. - P. 59 - 81.

3. Btaszak M. Bi-Hamiltonian dynamical systems. - New York: Springer, 1998.

4. Bogolubov N. N. (Jr. )., Prykarpatsky A. K. Complete integrability of the nonlinear Ito and Benney-Kaup systems: gradient algorithm and Lax representation // Theor. and Math. Phys. - 1986. - 67, № 3. - P. 586-596.

5. Chen Tao, Zhu Li-Li, Zhang Lei. The generalized Broer-Kaup-Kupershmidt system and its Hamiltonian extension // Appl. Math. Sci. - 2011. - 5, № 76. - P. 3767-3780.

6. Coddington E. A., Levinson N. Theory of differential equations. - New York: McGraw-Hill, 1955.

7. Faddeev L. D., Takhtadjan L. A Hamiltonian methods in the theory of solitons. - New York; Berlin: Springer, 2000.

8. Foursov M. V. On integrable coupled Burgers-type equation // Phys. Lett. A. - 2000. - 272. - P. $57-64$.

9. Fritz AJ., Troth B. Derivation of the Leroux system as the hydrodynamic limit of a two-component lattice gas // Communs Math. Phys. - 2004. - 249, Issue 1. - P. 1-27.

10. Gelfand I. M., Dickey L. A. Integrable nonlinear equations and Liouville theorem // Funct. Anal. and Appl. - 1979. 13. - P. 8-20.

11. Gelfand I. M., Dickey L. A. The calculus of jets and nonlinear Hamiltonian systems // Funct. Anal. and Appl. 1978. - 12, Issue 2. - P. $81-94$.

12. Gelfand I. M., Dickey L. A. A Lie algebra structure in a formal variational calculation // Funct. Anal. and Appl. 1976. - 10, Issue 1. - P. 16-22.

13. Gelfand I. M., Dickey L. A. The resolvent and Hamiltonian systems // Funct. Anal. and Appl. - 1977. - 11, Issue 2. P. $93-105$.

14. Gelfand I. M., Manin Yu. I., Shubin M. A. Poisson brackets and the kernel of the variational derivative in the formal calculus of variations // Funct. Anal. and Appl. - 1976. - 10, Issue 4. - P. $274-278$.

15. Godbillon C. Geometri differentielle et mecanique analytique. - Paris: Hermann, 1969.

16. Ibragimov N. H., Shabat A. B. Infinite Lie-Backlund algebras // Funct. Anal. and Appl. - 1980. - 14. - P. 313 - 315.

17. Kaplanski I. Introduction to differential algebra. - New York, 1957.

18. Kupershmidt B. A. Dark equations // J. Nonlinear Math. Phys. - 2001. - 8, № 3. - P. $363-445$.

19. Kupershmidt B. A. Mathematics of dispersive water waves // Communs Math. Phys. - 1985. - 99. - P. 51 - 73.

20. Lax Peter D. Almost periodic solutions of the KdV equation // Source: SIAM Rev. - 1976. - 18, № 3. - P. 351 - 375.

21. Lions J. L. Quelgues methodes de resolution des problemes aux limites non lineaires. - Paris: Dunod, 1969. 
22. Ma W. X. A hierarchy of of coupled Burgers systems possessing a hereditary structure // J. Phys. A: Math. and Gen.1993. - 26. - P. L1169-L1174.

23. Mikhailov A. V., Shabat A. B., Yamilov R. I. The symmetry approach to the classification of nonlinear equations. Complete list of integrable systems // Russ. Math. Surv. - 1987. - 42, № 4. - P. 1 - 63.

24. Mikhailov A. V., Shabat A. B., Yamilov R. I. Extension of the modul of invertible transformations. Classification of integrable systems // Communs Math. Phys. - 1988. - 115. - P. 1 - 19.

25. Theory of solitons: the inverse scattering method / Ed. S. P. Novikov. - Springer, 1984.

26. Olver P. Applications of Lie groups to differential equations. - Second ed. - New York: Springer-Verlag, 1993.

27. Prykarpatsky A. K., Artemovych O. D., Popowicz Z., Pavlov M. V. Differential-algebraic integrability analysis of the generalized Riemann type and Korteweg-de Vries hydrodynamical equations // J. Phys. A: Math. Theor. - 2010. 43. - P. $295-205$.

28. Prykarpatsky Y. A., Artemovych O. D., Pavlov M., Prykarpatsky A. K. The differential-algebraic and bi-Hamiltonian integrability analysis of the Riemann type hierarchy revisited // J. Math. Phys. - 2012. - 53; doi 10.1063/1.4761821.

29. Prykarpatsky Y. A., Artemovych O. D., Pavlov M., Prykarpatsky A. K. The differential-algebraic integrability analysis of symplectic and Lax type structures related with the hydrodynamic Riemann type systems // Repts Math. Phys. 2013. - 71, № 3. - P. 305-351.

30. Prykarpatsky Y. A. Finite dimensional local and nonlocal reductions of one type of hydrodynamic systems // Repts Math. Phys. - 2002. - 50, № 3. - P. 349-360.

31. Prykarpatsky A., Mykytyuk I. Algebraic integrability of nonlinear dynamical systems on manifolds: classical and quantum aspects. - Netherlands: Kluwer Acad. Publ., 1998.

32. Ritt J. F. Differential algebra // AMS-Colloq. Publ. - New York: Dover Publ., 1966. - 33.

33. Shubin M. Lectures on mathematical physics. - Moscow: Moscow State Univ., 2001.

34. Sokolov V. V., Wolf T. Classification of integrable polynomial vector evolution equations // J. Phys. A: Math. and Gen. - 2001. - 34. - P. $11139-11148$.

35. Prykarpatski A., Soltanov K., Özçă̆ E. Differential-algebraic approach to constructing representations of commuting differentiations in functional spaces and its application to nonlinear integrable dynamical systems // Commun. Nonlinear Sci. Numer Simulat. - 2014. - 19. - P. $1644-1649$.

36. Tasso H. Hamiltonian formulation of odd Burgers hierarchy // J. Phys. A: Math. and Gen. - 1996. - 29. - P. 7779 7784.

37. Tsuchida T., Wolf T. Classification of polynomial integrable systems of mixed scalar and vector evolution equations. I // J. Phys. A: Math. and Gen. - 2005. - 38. - P. $7691-7733$.

38. Whitham G. B. Linear and nonlinear waves. - John Wiley \& Sons Inc., 1974.

39. Wilson G. On the quasi-Hamiltonian formalism of the KdV equation // Phys. Lett. - 1988. - 132, № 8/9. - P. 445 - 450.

Received 06.01 .14 